\title{
Posibilidades Y LímITES DEL CRECIMIENTO Y DESAROLLO ECONÓMICO-SOCIAL EN PAÍSES DE EUROPA, ASIA, ÁFRICA Y AMÉRICA LATINA
}

\author{
POSSIBILITIES AND LIMITS OF THE GROWTH AND SOCIAL ECONOMIC \\ DEVELOPMENT IN THE COUNTRIES OF EUROPE, ASIA, AFRICA \\ AND LATIN AMERICA \\ Esperanza Bonilla Olano*
}

Recibido: 18 de agosto de 2017

Aceptado: 14 de noviembre de 2017

\section{Resumen}

Desde la perspectiva de las teorías de la maldición de los recursos y el neoextractivismo y de sus antecedentes teóricos, el objetivo del artículo es contrastar estos referentes con las realidades de algunos países que fueron consultadas en estudios realizados por investigadores interesados en la temática en cuestión. Como resultado, se identifican experiencias con efectos favorables y desfavorables en términos de estímulo o retraso al crecimiento y desarrollo en países de Europa, Asia, África y América Latina, cuyo modelo económico se ha sustentado en las actividades extractivas y de producción de materias primas, orientadas al comercio exterior.

Palabras clave: recursos naturales, la maldición de los recursos, extractivismo, neoextractivismo.

\begin{abstract}
From the perspective of the curse of resource theories and neo-extractivism and its theoretical background, the objective of the article is to highlight these referents with the realities of some countries interviewed in studies directed by researchers interested in the topic in question. As a result, experiences with favorable and unfavorable effects are identified regarding stimulation or delay to growth and development in countries of Europe, Asia, Africa, and Latin America, which economic model has based on extractive activities and production of raw materials, oriented to foreign trade.
\end{abstract}

Keywords: natural resources, the curse of resources, extractivism, neo-extractivism.

* Economista, Docente-Investigadora del Grupo Desarrollo y Equidad de la Facultad de Economía. Fundación Universidad América, Bogotá, Colombia. ORCID: https://orcid.org/0000-0003-1699-716X. esperanza.bonilla @ profesores.uamerica.edu.co 


\section{INTRODUCCIÓN}

La situación de muchos países subdesarrollados ${ }^{1}$ evidencia altos niveles de pobreza a pesar de poseer abundante riqueza expresada en recursos naturales; esta realidad ha originado que estudiosos e investigadores de la temática del desarrollo busquen explicaciones teóricas, más allá de las tradicionales u ortodoxas. Entre estas vale mencionar la sustentada por el liberalismo económico, la cual afirma que el mercado conducido por una "mano invisible" regula la economía y que gracias al manejo monetario y fiscal prudente se mantiene el equilibrio macroeconómico. Bajo esta concepción, es la austeridad pública la que contribuye a mantener dentro de los rangos determinados por las autoridades que fijan la política en cada país, los principales indicadores macroeconómicos, para que no sobrepasen sus metas.

Colombia, como la mayoría de países latinoamericanos, deriva sus ingresos principalmente por exportaciones de la producción de bienes primarios y del sector minero energético. Por tal razón, en este estudio se abordan las teorías de la maldición de los recursos y la neoextractivista como marcos de referencia para responder a los siguientes interrogantes: ¿La abundancia de recursos naturales, es realmente una maldición? ¿Es necesario replantear el postulado de que el crecimiento del país debe estar substancialmente asociado a la explotación minero-energética y a la exportación de otras materias primas provenientes del sector primario de la economía? ¿Los modelos extractivista y neoextractivista conducen al desarrollo del país?

Se hace una referencia conceptual y se contextualiza históricamente el surgimiento de las teorías de la maldición de los recursos naturales y neoextractivista, por tratarse de dos enfoques que tienen sus coincidencias y que hoy, de manera recurrente, los toman investigadores y estudiosos del crecimiento y del desarrollo como referentes. Asimismo, se reseñan experiencias de países que a pesar de poseer abundantes recursos naturales no han superado las condiciones de atraso o donde ni siquiera esta fortaleza se ve reflejada en mejores indicadores de redistribución de ingreso y bienestar de su población. Y otras más exitosas, que reflejan cómo el poseer estos recursos y el manejo que han hecho de los ingresos generados sí les ha permitido superar condiciones de subdesarrollo y avanzar no solamente en el crecimiento del PIB, sino por la senda del desarrollo expresado en mejoramiento de la calidad de vida de la población.

\section{ANTECEDENTES TEÓRICOS DE LA ESPECIALIZACIÓN EN LA EXPLOTACIÓN Y EXPORTACIÓN DE LOS RECURSOS NATURALES}

\section{Los postulados clásicos y neoclásicos del comercio internacional}

Adam Smith, David Ricardo y J.S. Mill fueron los pioneros en el desarrollo de la visión clásica del comercio internacional, afirmando que éste es una de las vías mediante la cual los países logran expandir sus mercados internos, y al hacerlo, crecer más rápidamente.

Smith, en su famosa obra Una investigación sobre la naturaleza y causas de la riqueza de las naciones (Smith, 2011), le atribuye al comercio internacional un importante papel en el crecimiento de los países. Plantea que una vez se ha incrementado la productividad en el interior de los países por efecto de la división del trabajo y la aplicación de nuevas tecnologías en los procesos productivos, se generan excedentes que no son absorbidos por los mercados internos y en consecuencia se les deben buscar espacios más allá de los límites nacionales, con el fin de que el mercado externo contribuya al crecimiento. Los bienes para exportar en que debe especializarse cada país, son aquellos en los cuales tenga ventaja competitiva absoluta (Smith) o comparativa relativa (Ricardo), derivada

${ }^{1}$ Para unos, en vías de desarrollo, para otros, países emergentes. 
de unos costos de producción más bajos que en el resto de naciones. La fuente de esta ventaja es la dotación de recursos humanos y materiales con que se cuente. Este postulado se aplicaría a los países latinoamericanos en donde los recursos naturales tales como petróleo, carbón, oro, y otros son abundantes. A su vez, es ventajoso adquirir en el exterior los bienes que resulta más costoso producir internamente. Desde la mirada de Mill, la ventaja está soportada en unos costos de oportunidad más bajos. Posteriormente, los considerados exponentes del neoclasicismo, Heckscher y Ohlin, a comienzos de los años setenta complementaron el postulado clásico atribuyéndole a la abundancia o escasez de factores, las ventajas o desventajas comparativas de un país frente al resto del mundo.

De los planteamientos anteriores se infiere que el comercio internacional está en la capacidad de generar beneficios de crecimiento y desarrollo porque, además, incentiva el cambio tecnológico para aumentar la productividad laboral ampliando la capacidad de los países para insertarse en los mercados internacionales, lo cual quiere decir que éstos contribuyen a su vez a expandir los mercados internos, a la especialización de la producción y a la profundización de la división internacional del trabajo (Puyana, 2017). Vale la pena tener en cuenta que en el caso de los países subdesarrollados, su estructura productiva se ha determinado teniendo en cuenta las demandas externas de bienes y servicios, lo cual ha ocasionado que a través del tiempo se privilegie la extracción y explotación de fuentes primarias, relegando el desarrollo industrial y el fortalecimiento del mercado interno.

\section{El estructuralismo y el deterioro de los términos de intercambio}

La teoría conocida como estructuralista-cepalina corresponde a un desarrollo efectuado por R. Prebisch y Paul Singer en la década de los cincuenta (Rodríguez, 2006). En su texto El desarrollo económico de la América Latina y algunos de sus principales problemas (1949), Prebisch, atribuye los problemas que afectan a la mayoría de países de América Latina, al hecho de que adoptaron la senda de la especialización en producción de materias primas, dándole una importancia marginal a la industrialización, como apuesta para lograr crecimiento y desarrollo de sus economías (Prebisch, 1949).

El mismo Prebisch, apoyado en evidencia empírica, analizó las relaciones económicas entre los países desarrollados, a los que denominó del centro, y los de la periferia o subdesarrollados. Como resultado de sus análisis encontró que estas relaciones se traducían en un deterioro de los términos de intercambio de los países periféricos. En consecuencia, cuestionó el planteamiento de los clásicos acerca de los beneficios que la división internacional del trabajo y las ventajas comparativas aportaban a los países de menor desarrollo (periféricos), debido a que los países del centro se caracterizaban por tener un importante progreso técnico, diversificación de sus economías, alta productividad y producciones con mayor valor agregado, mientras que los periféricos, al especializarse en la producción y exportación de bienes primarios con mínima o nula transformación, sin mayor valor agregado y con baja productividad, se beneficiaban mínimamente de sus intercambios comerciales. La condición de ser exportadores de materias primas y bienes primarios para satisfacer la demanda de los países de mayor desarrollo, impuesta por la división internacional del trabajo a los países subdesarrollados, llevó no solamente a sustentar la teoría del deterioro de los términos de intercambio de Prebisch y Singer, sino la del desarrollo desigual y la dependencia, expuesta por Furtado y Cardozo.

Otro aspecto negativo para que la especialización en exportar materias primas no sea favorable al país propietario de los recursos naturales, es el hecho de que la explotación y comercialización de dichos recursos se entrega en concesión a las multinacionales (Prebisch, 1949). Además, vale mencionar que a estas empresas las legislaciones nacionales les otorgan privilegios tributarios y facilidades para enviar las utilidades a sus países de origen sin mayor exigencia de reinvertir parte de éstas en el país donde operan. 


\section{La teoría de la maldición de los recursos. Concepto y contexto}

En la en la literatura económica, el término 'maldición de los recursos' se utiliza para connotar el impacto negativo en el desarrollo y el crecimiento económico como consecuencia de la dependencia de los recursos naturales (Oxfam, 2010). Quien primero utilizó la expresión fue Richard Auty (Mikesell y Raymond, 1998), "para describir cómo los países ricos en recursos naturales no podían usar esa riqueza para impulsar sus economías y cómo, en contra de toda intuición, estos países tuvieron un crecimiento económico menor que los países sin una abundancia de recursos naturales”.

Posteriormente, autores reconocidos como Jeffrey Sachs y Andrew Warner retomaron esta teoría para encontrar una relación entre crecimiento y abundancia de recursos y, a partir de entonces, ésta se convirtió para algunos en referente analítico que permite sustentar por qué algunos países, a pesar de disponer de abundante riqueza en términos de recursos naturales, se mantienen en el atraso; "en contra de toda lógica, la explotación de estos recursos está vinculada en muchos casos a la pobreza, la desigualdad, los servicios públicos de baja calidad, y el retraso en el crecimiento económico" (Itriago, 2009 p.1). La explicación del porqué de este hecho tan contradictorio se fundamente en lo que se denomina la lógica de desplazamiento y se expresa de la siguiente manera: la explotación de recursos naturales desplaza a la actividad manufacturera x. La actividad x impulsa el crecimiento. Por lo tanto, los recursos naturales dañan el crecimiento (Sachs y Warner, 2001). Sachs y Warner identifican a x con las actividades manufactureras comerciadas.

No obstante lo expuesto por Jeffrey Sachs, los recursos naturales deberían considerarse como una fortaleza de los países que los poseen y como tal, fuente de ingresos para procurar su crecimiento desarrollo y bienestar, y en ese sentido, desde la mirada de la teoría de la maldición de los recursos, esta situación encierra una paradoja. En efecto, la mayoría de los países con dotación de recursos naturales como petróleo o minerales, tienden a tener peores resultados en sus indicadores de crecimiento y desarrollo que los que no los tienen. Entre las razones principales que se aducen, están: la generación de la enfermedad holandesa ${ }^{2}$ y la vulnerabilidad a la que queda expuesta una economía, al ser muy dependiente de los ingresos por exportación de recursos minero-energéticos y provenientes del sector primario, cuyos precios son muy volátiles en el mercado internacional. Tal como lo expresa (Morales, 2012), los países de África, América y Asia tienen una excesiva dependencia de los productos primarios, lo que hace sus economías fluctuantes en función de los precios de sus principales recursos.

A lo anterior hay que adicionar el hecho que se presenta con los Estados que son muy dependientes de los ingresos de los recursos naturales cuando en épocas de precios altos estos les generan ingentes recursos, pero no los reinvierten en proyectos que redunden en bienestar y calidad de vida para la población, v. gr. educación, salud e infraestructura vial, o en realizar inversiones que generen una transformación del aparato productivo, orientada al desarrollo industrial, tecnológico y a la innovación.

Itriago (2009) amplía la explicación en el sentido de que la explotación de la mayoría de recursos naturales requiere de tecnología costosa, de la cual no disponen los países que poseen el recurso, y es en este insumo donde se ubica la mayor parte de la inversión extranjera, en el caso colombiano el 41\%. Para los Estados, los ingresos por la venta de estas materias primas en el mercado internacional son superiores al compararlos con los de otras actividades industriales, situación que produce una concurrencia de intereses del sector público, el Estado y las empresas multinacionales, privilegiando esta actividad del sector primario sobre estímulos al desarrollo industrial.

\footnotetext{
${ }^{2}$ Se denomina 'enfermedad holandesa' al fenómeno de expansión del ingreso de divisas por auges en la exportación de recursos naturales (petróleo y minerales) que, sin las medidas adecuadas, genera revaluación de la moneda local y contracción y pérdida de empleos en otros sectores productivos.
} 
Una debilidad asociada a los productos primarios es que tal y como se exportan no tienen un apreciable valor agregado, y según lo plantea la visión heterodoxa, al ser canjeados en el mercado internacional por bienes industriales, generan una relación desventajosa en términos de intercambio para los países exportadores de dichos bienes, como el caso de Venezuela con el petróleo o de Irán con el uranio. De acuerdo con (Prebisch, 1986), dada la especialización de los países en desarrollo en la producción de materias primas y alimentos, la inestabilidad de los precios de estos productos llevaría al deterioro de los términos de intercambio de estos países de manera secular.

Según Carrera (2017), la maldición de los recursos tiene expresiones económicas debido a la variabilidad de precios: una ocurre cuando éstos son altos y se corre el riesgo de sufrir la enfermedad holandesa; otra es de gestión macroeconómica, reflejada en la baja utilización de los recursos en programas de salud, infraestructura, educación, investigación e innovación. Otro problema estructural que se evidencia es el de la apropiación de rentas por parte de particulares y evasión tributaria. A nivel político se generan conflictos bélicos por el control de los recursos naturales, lo que lleva a sistemas autoritarios.

\section{El extractivismo y el neoextractivismo}

El extractivismo es una orientación del desarrollo económico de un país que privilegia la explotación intensiva y venta al exterior de los recursos naturales con bajo nivel de procesamiento o valor agregado. En el siglo XX, el extractivismo contribuyó a acentuar la división internacional del trabajo y la especialización, de tal manera que los países desarrollados se convirtieron en demandantes de materias primas provenientes de los países subdesarrollados, lo cual ha proyectado en el tiempo una gran dependencia de la extracción de recursos naturales y materias primas provenientes del sector primario, concebidas como generadoras de crecimiento en dichos países. Esta división del trabajo ha determinado la estructuración del sistema capitalista mundial (Colectivo Casa, 2015).

Según los teóricos del estructuralismo y la dependencia, los efectos del extractivismo en las economías de los países especializados en recursos naturales se expresan principalmente en aspectos como: la alta dependencia de los compradores en los mercados externos; la inequidad en el intercambio comercial entre materias primas y productos transformados, debido a que estos últimos son mejor remunerados; y el mal manejo de las y rentas recibidas por el Estado, que en muchos casos no cumple con la labor de redistribución que debería cumplir. Adicionalmente, cuando los precios internacionales son favorables, se generan presiones inflacionarias por el elevado volumen de divisas que ingresan, y también, hay una concentración de la inversión extranjera directa en estas actividades en menoscabo del desarrollo de otras de carácter industrial. Por otra parte, la ejecución inadecuada de la explotación de estos recursos conlleva impactos medioambientales negativos.

La esencia conceptual del denominado neoextractivismo hace referencia a las políticas que fortalecen el papel del Estado en la explotación de recursos y el manejo de los ingresos por exportaciones, afirmando que a dicha actividad sería consustancial un efecto redistributivo (Gudynas, 2010). Este modelo es estimulado principalmente cuando se presentan altos precios internacionales de los bienes primarios favorecidos por la demanda de los países asiáticos, principalmente China.

Desde los años noventa, en América Latina se ha privilegiado un modelo de desarrollo exógeno extractivista, que ubica como estratégico el comercio internacional sustentado en la exportación de recursos naturales. Tal modelo está inserto en el esquema neoliberal, según Azamar y Ponce (2015), quienes sostienen que "el crecimiento económico debe apoyarse en una mayor apertura comercial para lograr un mejor aprovechamiento de la especialización, con respecto a las ventajas comparativas por país, y en un proceso de industrialización más intensivo de los recursos naturales" (p.186). 
En Latinoamérica: Chile y Argentina fueron los pioneros en implementar el proyecto neoextractivista en el marco del ajuste estructural y de liberalización de su economía, llevado a cabo a partir de la década del setenta. Posteriormente, en los noventa, se retomó este enfoque en el resto de América Latina.

Tanto el extractivismo como el neoextractivismo tienen en común que sustentan el desarrollo del comercio exterior en la exportación de recursos naturales y materias primas con exigua transformación. La diferencia entre las dos orientaciones radica en que la concepción neoextractivista le da una importancia primordial a la participación del Estado en la actividad, a través de empresas estatales y de concesiones a empresas extranjeras (multinacionales). Esta modalidad le garantiza la obtención de ingresos al Estado (Carrasco y del Hoyo, 2010). La actividad extractivista, requiere grandes inversiones, de ahí que la Inversión Extranjera Directa (IED) que llega a los países latinoamericanos se ubique en estos subsectores en una importante proporción; en el caso de Colombia, entre el 2011 y el 2014, la IED en actividades extractivas representó alrededor del $51 \%$ de la IED total que recibió el país.

Estudiar la extracción de recursos naturales y la producción de otras materias desde la perspectiva de la teorización del comercio internacional, implica abordar la estructura del comercio mundial y ubicar estas actividades dentro de la división internacional del trabajo y su articulación con las cadenas globales de valor (Gudynas, 2010). En este contexto, Gudynas (2010) hace la siguiente caracterización de la forma que ha adquirido el extractivismo en América Latina, en su articulación con la economía global, dice:

Los procesos extractivistas contemporáneos en Latinoamérica se caracterizan por ser economías de enclave, localizadas en regiones específicas con presencia de recursos naturales, como metales, petróleo, gas natural o agua; o bien en la superficie, como el monocultivo agroindustrial, la minería a cielo abierto y los recursos forestales, entre otras. En estas economías, generalmente, se explota más de un recurso -orientado a las necesidades del mercado internacional-, y son prácticamente nulos los encadenamientos productivos con la economía local. La mano de obra en los negocios extractivos está mal pagada y realiza actividades de alto riesgo, que ponen en juego su vida y su salud. La mayoría de estos proyectos no tienen una duración mayor a treinta años, ya sea por el agotamiento del recurso o de las propias tierras; además, no dejan ningún provecho a la población, fuera de las contribuciones fiscales a los países en donde estos enclaves se desarrollan.

Contrastes entre teoría y evidencias. No obstante que no existe mayor evidencia empírica sobre el tema, el asunto de si la dotación de recursos naturales y la especialización en su explotación y exportación trae o no crecimiento y desarrollo a los países, es muy discutido. Las siguientes experiencias recogidas de autores que han trabajado el tema, confirman lo polémico que puede ser.

Experiencias favorables. En América Latina, en el pasado reciente, Ecuador se destaca por haber utilizado la bonanza petrolera de la década pasada en inversiones en infraestructura vial y eléctrica y en educación, contribuyendo con ello a mejorar la calidad de vida tanto de los habitantes rurales como urbanos.

Un caso que vale la pena resaltar es el de Namibia (Carotti, 2016), país que se independizó de Suráfrica en 1990 y que ha logrado importantes progresos, no obstante tener una fuerte dependencia de la minería, que le aporta el 8\% de su PIB y alrededor del $50 \%$ de sus exportaciones. Por sus abundantes yacimientos de diamante, es uno de los principales exportadores mundiales de gemas; es el cuarto productor mundial de uranio y posee también yacimientos de zinc y oro. Entre el 1991 y 2014 mantuvo un crecimiento promedio de 4.2 \% y a partir del 2015, lo elevó a 4.5 \%, a pesar de que el descenso en el mercado mundial de los precios de las materias primas también lo 
afectó. Muchos de sus avances se han soportado en su exitosa política educativa y la inversión de recursos en salud; en efecto, en diez años redujo los casos de malaria en un $97 \%$ y disminuyó la pobreza extrema en un $20 \%$.

En la Unión Europea, Suecia es el país más representativo en la extracción de minerales y metales. Ha basado su desarrollo industrial, y simultáneamente sus encadenamientos productivos al interior del país, en la acumulación de capital, lo cual le ha permitido hacer prosperar su sector minero. A partir de la renovación tecnológica este país ha logrado aumentar su productividad pasando de exportar 8 millones de toneladas de recursos naturales al año hace un siglo, a 80 millones en la actualidad (Carotti, 2016).

Como estrategia económica central, estas naciones permitieron que sobre la base de la exportación de productos primarios se dieran procesos de acumulación de capital que desarrollaron el aparato productivo manufacturero.

Otra evidencia de cómo los recursos naturales sí pueden fundamentar el desarrollo es el caso de Noruega, tercer mayor exportador de petróleo en el mundo, mayor abastecedor de gas natural a los países de Europa Occidental y mayor exportador de pescado. En definitiva, en este país nórdico, el 80\% de las exportaciones provienen de sus recursos naturales: hidrocarburos, pescado, metales y madera. La industria petrolera noruega se destaca a nivel internacional por estar a la vanguardia en la utilización de tecnologías que protegen el medio ambiente. Y además, su política de redistribución de los ingresos cuenta con un programa, abanderado en Europa, en el que parte de las ganancias del petróleo van destinadas a un fondo de inversión que le garantice recursos a las futuras generaciones. Este fondo de inversión es el cuarto más grande a nivel mundial.

Otra experiencia exitosa en el aprovechamiento de las ganancias provenientes de los recursos naturales, es la observada en la República de Botswana, en África, que logró desarrollar unas instituciones adecuadas y sólidas por medio de una dirigencia comprometida en generar desarrollo, la cual administró los recursos naturales de tal forma que diversificó la economía evitando la dependencia excesiva del sector minero. Por otra parte, alcanzó un significativo recaudo fiscal y ha separado el gasto público de la industria extractiva, para así destinar parte de los ingresos por exportaciones de recursos naturales a las futuras generaciones.

Carrera (2017) cita otros casos de países que se han hecho prósperos gracias, en parte, a su dotación de recursos naturales: Argentina, Uruguay, Canadá, Australia, Nueva Zelanda Emiratos Árabes y Arabia Saudí.

Experiencias desfavorables. Nigeria es el caso típico de África que percibe gran cantidad de recursos derivados del petróleo y tiene uno de los indicadores de pobreza y atraso más altos del continente; igualmente Angola, un país con grandes reservas petroleras y minerales (diamantes) que debido al auge del petróleo creció casi un $20 \%$ entre los años 2005 y 2008, sin embargo, sufre un pobre desarrollo social y es un país donde la mayoría de la población vive de una agricultura de subsistencia y con menos de un dólar al día. (Suarez, 2013). Si se compara el gasto público de Angola con la media del resto de países de renta baja, dicho gasto está por encima y sin embargo, como ya se dijo, este gasto no se ha visto reflejado en una mejor calidad de vida de sus habitantes.

Así mismo, países latinoamericanos exportadores de minerales y otros productos agrícolas, como por ejemplo banano y cacao, no presentan precisamente los mejores resultados en cuanto a redistribución del ingreso y bienestar.

Resultados tan disímiles en diferentes países sugieren buscar explicaciones complementarias y contextualizadas en cada caso. 
En el siguiente gráfico, se hace una relación entre el coeficiente de Gini y el índice de desarrollo humano (IDH) ${ }^{3}$, con el fin de obtener a través de estos dos indicadores una visión transversal, (año 2016) del nivel de distribución del ingreso y del estado en materia de desarrollo de los países a que hace referencia este artículo.

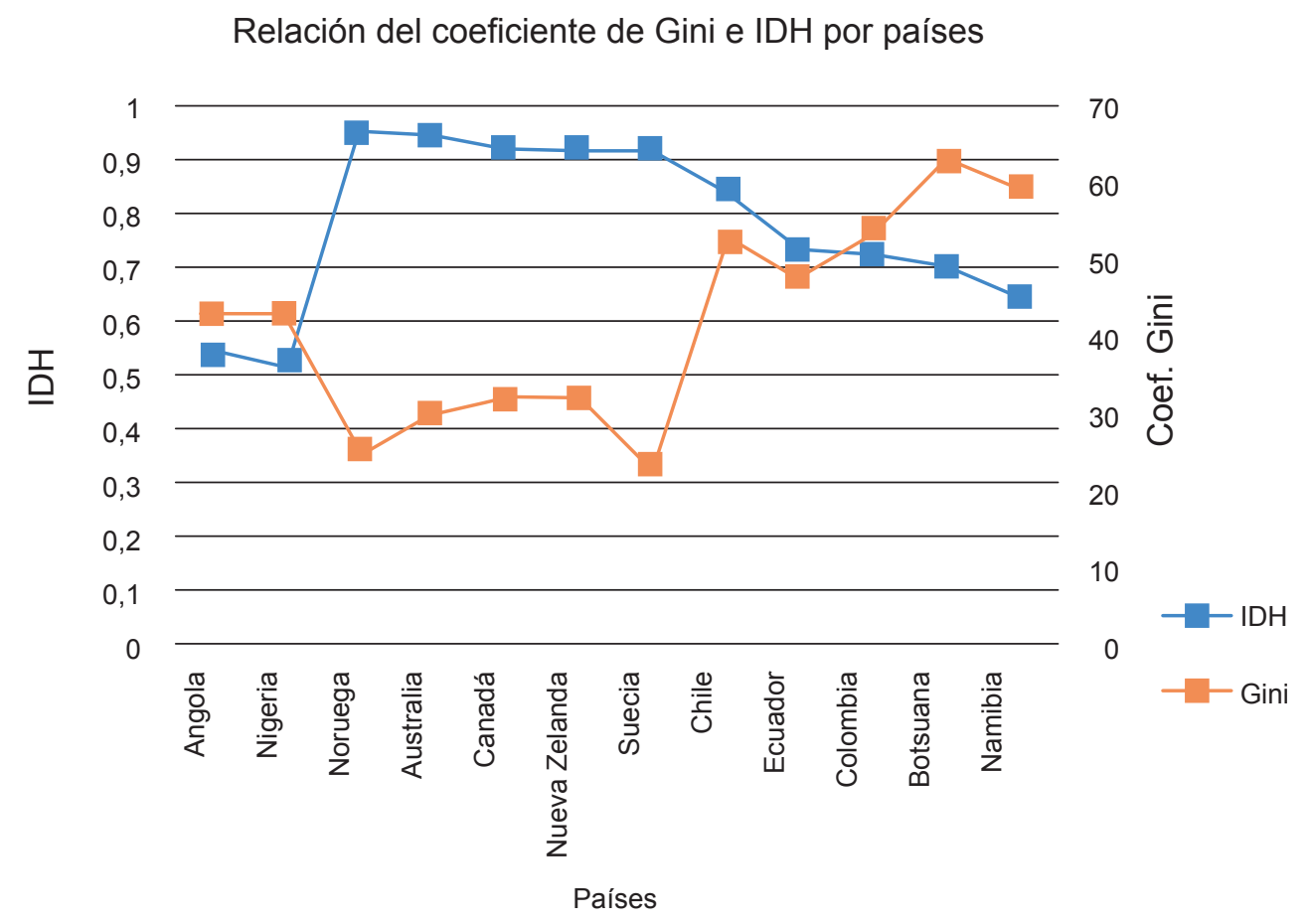

Figura 1. Relación del coeficiente de Gini, Índice de Desarrollo Humanos por países

Fuente: Elaboración propia, con base en datos del Banco Mundial.

En Angola y Nigeria, países africanos que basan su crecimiento en la explotación de recursos naturales, se observa una alta concentración del ingreso y un bajo IDH. Es decir, las actividades extractivas no han beneficiado su desarrollo. En los países de un alto nivel de desarrollo como Noruega, Australia, Canadá, Nueva Zelanda y Suecia se evidencia una favorable distribución del ingreso y un alto IDH, lo cual valida lo descrito anteriormente en este artículo. En cuanto a los países latinoamericanos incluidos en este análisis, las cifras indican una alta concentración del ingreso y, a pesar de esto, un IDH favorable, a excepción de Colombia, cuyo coeficiente de Gini refleja una baja redistribución de ingreso y un IDH también relativamente bajo.

Ecuador es un caso interesante, como se mencionó con anterioridad, pues los recursos del petróleo fueron utilizados en inversiones en infraestructura, educación y salud, lo que se ve reflejado en el mejoramiento en la calidad de vida de sus habitantes, expresado en un IDH relativamente alto. Los casos de Botsuana y Namibia, no obstante mostrar unos altos coeficientes de concentración de ingreso, reflejan una mejora en su IDH, comparado con otros países africanos.

${ }^{3}$ El Índice de Desarrollo Humano (IDH) se creó para hacer hincapié en que la ampliación de las oportunidades de las personas debería ser el criterio más importante para evaluar los resultados en materia de desarrollo. El crecimiento económico es un medio que contribuye a ese proceso, pero no es un objetivo en sí mismo.

El IDH mide el progreso conseguido por un país en tres dimensiones básicas del desarrollo humano: disfrutar de una vida larga y saludable, acceso a educación y nivel de vida digno. 


\section{Conclusiones}

De acuerdo con los enfoques consultados, se infiere que hay aspectos controversiales. Si se toman como referentes los enfoques clásico y neoclásico, el extractivismo debe ser el camino que conduce al crecimiento económico, toda vez que, según sus planteamientos, la abundancia de recursos naturales le otorga ventaja comparativa a los países que disponen de estos recursos y ello los ha llevado a especializarse en su producción y en los mercados internacionales. A esta argumentación habría que objetarle que la mayoría de los recursos naturales son no renovables, como por ejemplo el petróleo, el carbón y el oro, entre otros, y que si los ingresos generados por su venta no se aprovechan y se orientan a inversiones en infraestructura y al incremento y diversificación de la capacidad productiva de los países, paradójicamente la abundancia de estos recursos perpetúa en el largo plazo condiciones de atraso y de no desarrollo.

Según las teorías estructuralistas y de la dependencia la especialización en recursos naturales ha llevado a los países en desarrollo a insertarse en la economía mundial a través de la división internacional del trabajo, marcándoles una especialización secular en la exportación de dichos recursos. Para los países subdesarrollados, seguir esta senda extractivista perpetúa en el tiempo escenarios de dependencia y atraso debido a que las condiciones asimétricas a que se ven sometidos en el intercambio comercial con países desarrollados amplía la brecha tecnológica, de productividad y de innovación, profundiza el deterioro ambiental y acrecienta las desigualdades con los países del centro, ahondando la heterogeneidad estructural y la vulnerabilidad de las economías subdesarrolladas. Todo lo anterior se constituye en una limitante para el crecimiento económico y desde luego para el desarrollo.

Según las corrientes de la maldición de los recursos naturales y neoextractivista la reprimarización que se está dando en la actividad económica, particularmente en países latinoamericanos, africanos y algunos asiáticos, profundiza la inserción de las economías latinoamericanas en el comercio internacional apoyándose en el capital extranjero, al abrir a las inversiones privadas sectores que antes no les era permitido. La tendencia actual es darle a las multinacionales cada vez mayor participación en la inversión en recursos que son considerados propiedad de la nación y cuya explotación corresponde a entes estatales, como son: las tierras baldías, el agua, la electricidad, el petróleo y el gas. Mientras que a dichas empresas transnacionales no se les exija cumplir con la responsabilidad social del pleno respeto al medio ambiente, a los derechos humanos y en general a la legislación de los países donde operan, y a reinvertir parte de sus utilidades en estos, su contribución al desarrollo será inverso y más aún si se le da la connotación de sostenible. Como lo expresa Raúl Prebisch, esta situación hace poco favorable la especialización en actividad extractiva de materias primas con destino al comercio internacional.

Una posición excesivamente radical sería plantear que los países que disponen de recursos naturales y los llevan a los mercados internacionales, al no derivar de esta actividad mayores beneficios en favor de mejorar la calidad de vida de sus habitantes deberían abandonarla.

Sin necesidad de abandonar la actividad extractiva por parte de los países periféricos, sí se requiere tomar medidas que impidan seguir profundizando el impacto negativo social y ambiental del extractivismo. Debido al agotamiento de los recursos no renovables, la continuidad del modelo extractivista se proyecta inviable, por tal razón se requiere hacer ajustes al modelo para modificar estructuras productivas que permitan diversificar el portafolio exportador incluyendo productos con alto valor agregado.

Teniendo en cuenta que Colombia, al igual que otros países latinoamericanos, ha fundamentado su actividad externa en la reprimarización de la producción minera liderada por el petróleo, el carbón y otros minerales y ello ha llevado al país, dentro de la división internacional del trabajo, a configurar una estructura productiva de baja complejidad, a tener un mercado interno débil y 
a depender del comercio exterior, se hace necesario una reorientación del modelo de desarrollo, soportada en abordajes teóricos que justifiquen de manera contextualizada la redistribución de los ingresos provenientes de las materias primas y su utilización eficiente para mejorar el desarrollo, tanto económico como social del país, tal como lo han logrado naciones como Suecia y Noruega.

La revisión del modelo extractivista requiere de una firme decisión política por parte de los gobiernos para plantear políticas que con su ejecución lleven al desarrollo de alternativas productivas frente a la vulnerabilidad que acarrea el sector externo soportado en el extractivismo.

Como se expresó en párrafos anteriores, dado que la explotación y venta de los recursos naturales se ha dejado en manos de empresas extranjeras multinacionales, es necesario diseñar un condicionamiento normativo para dichas empresas de tal forma que de sus utilidades netas sea invertido un porcentaje significativo que se vea reflejado en el desarrollo de los países propietarios de los recursos naturales.

\section{REFERENCIAS}

Carotti, M. (2016). ¿La "maldición de los recursos naturales" funciona en Noruega, Suecia y Namibia? Recuperado de http://martincarotti.blogspot.com.co/2016/07/recursos-naturales-maldicionu.html

Carrasco, I., y del Hoyo, J. (2010). Neoextractivismo. Recuperado de http://omal.info/spip. php?article 4847

Carrera, M. (2017). Globalizacion y entorno economico internacional (apuntes inéditos). Conferencias presentada en Fundación Universidad de América, Bogotá, Colombia.

Colectivo Casa. (2015). Extractivismo, dependencia y desarrollo. Recuperado de http://www.colectivocasa.org.bo/index.php?option=com_k2\&view=item\&layout=item\&id=140\&Itemid=124 sf

Gudynas, E. (2010). Si eres tan progresista ïpor qué destruyes la naturaleza? Neoextractivismo, izquierda y alternativas. Debate, 79, 61-81.

Itriago, D. (2009). Informe de Oxfam (Inédito).

Auty, R., y Mikesell, R. (1998). Sustainable development in mineral economies. Nueva York: Oxford University Press.

Morales, J. (2012). ¿Qué hay detrás de la Maldición de los Recursos Naturales? Estudio de Caso: los Paises Bajos. Madrid: Instituto Universitario de Desarrollo y Cooperación y Universidad Complutense de Madrid.

Internacional Oxfam. (2010). Informe Anual 2009-2010. Recuperado de https://www.oxfam. org/sites/www.oxfam.org/files/file_attachments/story/oxfam_internacional_-_informe_ anual_2009-2010_0.pdf\%0A

Ponce, J., y Azamar, A. (2015). El neoextractivismo como modelo de crecimiento en América Latina. Economía y Desarrollo, 154(1), 185-198..

Prebisch, R. (1949). El desarrollo económico de la América Latina y algunos de sus principales problemas. Desarrollo Económico, 26(103),479-502.

Prebisch, R. (1986). Notas sobre el intercambio desde el punto de vista periférico. Revista de la CEPAL, 28, 195-206. 
Puyana, A. (2017). El retorno al extractivismo en América Latina. ¿Ruptura o profundización del modelo de economía liberal y por qué ahora? Espiral, 14(69), 73-113.

Rodríguez, O. (2006). El estructuralismo latinoamericano. México: Siglo XXI Editores.

Sachs, J., y Warner, A. (2001). The curse of natural resources. European Economic Review, 45, 827838.

Smith, A. (2011). La riqueza de las naciones (Carlos Rodríguez Braun, trad.) España: Alianza.

Suárez, E. (2013). La maldición de los recursos naturales. El País. Recuperado de https://elpais. com/elpais/2013/11/01/3500_millones/1383289200_138328.html 